\title{
Further insights on dynamic morphological transitions in quasi-two-dimensional electrodeposition
}

\author{
M.-Q. Lòpez-Salvans, ${ }^{1}$ F. Sagués, ${ }^{1}$ J. Claret, ${ }^{1}$ and J. Bassas ${ }^{2}$ \\ ${ }^{1}$ Departament de Química-Física, Universitat de Barcelona, Martí i Franquès 1, 08028 Barcelona, Spain \\ ${ }^{2}$ Serveis Científico Tècnics, Universitat de Barcelona, Solé i Sabarís 1-3, 08028 Barcelona, Spain
}

(Received 8 May 1997)

\begin{abstract}
Dynamic morphological transitions in thin-layer electrodeposits obtained from copper sulphate solutions have been studied. The chemical composition of the electrodeposits indicates that they appear as a consequence of the competition between copper and cuprous oxide formation. In addition, the Ohmic control of the process is verified at initial stages of the deposit growth. At higher deposit developments, gravity-induced convection currents play a role in the control of the whole process and affect the position of these transitions. [S1063-651X(97)07411-4]

PACS number(s): 82.45. $+\mathrm{z}$, 81.10.Dn, 66.10.Ed, 81.15.Pq
\end{abstract}

\section{INTRODUCTION}

Dynamic morphological transitions are well known in quasi-two-dimensional electrodeposition experiments [1-7]. They consist in a sudden change of the characteristics of the deposit during growth, mainly its color and branching degree, which reproduces the geometry of the electrochemical cell. Thus the deposit appears divided into different consecutive regions separated by transition lines. This phenomenon has been observed under different experimental conditions, using purely binary electrolytes [1-5,7], as well as in the presence of added inert electrolytes [6] or nondepositing species [7] in the solution.

It is widely accepted that the changes experimented in the growing deposit are due to a pronounced modification in the depositing conditions as a consequence of the interaction of the growing deposit with chemical fronts advancing from the anode towards the cathode. In particular, different transitions, associated with different chemical fronts, have been reported in zinc electrodeposition experiments from a zinc sulphate solution [7]: a basic front, an impurity (alcaline ion) free front, and also a concentration front related to an anodic convection roll induced by gravity effects arising from density gradients. On the other hand, an acid front also has been identified as responsible for the transitions observed using copper sulphate solutions $[2,3,6]$. This front is formed at the anode due to the hydrolysis of copper ions resulting from the anode dissolution [2]. In nonbinary electrolyte experiments, namely, a copper sulphate solution with small amounts of an inert electrolyte, typically any alcaline sulphate [6], a transition due to an alcaline ion free front is also observed in addition to the mentioned acid transition.

However, in spite of some more or less widely spread agreement concerning the origin of this phenomenon, some aspects remain uncertain and deserve a more detailed analysis. In relation to the transitions observed with pure copper sulphate solutions, the color change has been ascribed to the presence of cupric oxide in the first deposit structure. However, no experimental proof has been presented so far supporting this conjecture [1,2]. On the other hand, assuming an Ohmic control of the whole process, the position of this transition has been normally justified from the relation of the mobility of the ionic species responsible for the front (hydro- gen ion) and that of the anion that is assumed to limit the growth velocity of the deposit $[2,3]$. Nevertheless, in most cases, the values of the ion mobilities have been taken at infinite dilution and, moreover, the experimental conditions under which the Ohmic regime is fulfilled have not been accurately verified.

The primary aims of this paper are, first, to discuss the chemical changes associated with dynamic transitions in binary copper sulphate solutions, second, to verify the experimental conditions and also the growth stages in which the Ohmic regime is mostly achieved and, finally, to discuss the position of the morphology transition in terms of real ion mobility values.

\section{EXPERIMENT}

The electrochemical cell for quasi-two-dimensional electrodeposition consists of two 5-mm-thick glass plates separated by the electrodes. The cell gap is determined by the diameter of the copper wires acting as electrodes. Most of experiments were conducted in a cell enclosing two parallel electrodes separated by a distance of $4 \mathrm{~cm}$.

Electrodeposits were grown under a constant potential difference between the cathode and the anode. Potentials were applied by means of a Premium SR 120 or a Sabar power supply. The current and cathode potential measurements were conducted with a Hewlett Packard 34401A multimeter and processed in a personal computer. Conductivity measurements were performed with a Radiometer CDM3 conductivity meter under the same experimental conditions as for the electrodeposition experiments. $p \mathrm{H}$ values were determined by means of a Metrohm $605 p \mathrm{H}$ meter. The image recording and analysis system has been described elsewhere [6]. Experiments were carried out at room temperature.

$\mathrm{X}$-ray-powder diffraction measurements were performed using a Bragg-Brentano $\Theta / 2 \Theta$ diffractometer Siemens D500 under the following experimental conditions: a $\mathrm{Cu} K \alpha$ radiation $(\lambda=1.5418 \AA)$ at $40 \mathrm{kV}$ and $30 \mathrm{~mA}$, a secondary graphite monochromator, a divergence slit of $1^{\circ}$, and a receiving slit of $0.05^{\circ}$. In order to prepare the samples, electrodeposits obtained in a series of experiments using electrochemical cells with thickness of $300 \mu \mathrm{m}$ were collected, dried, and stored under argon atmosphere. Then the sample 

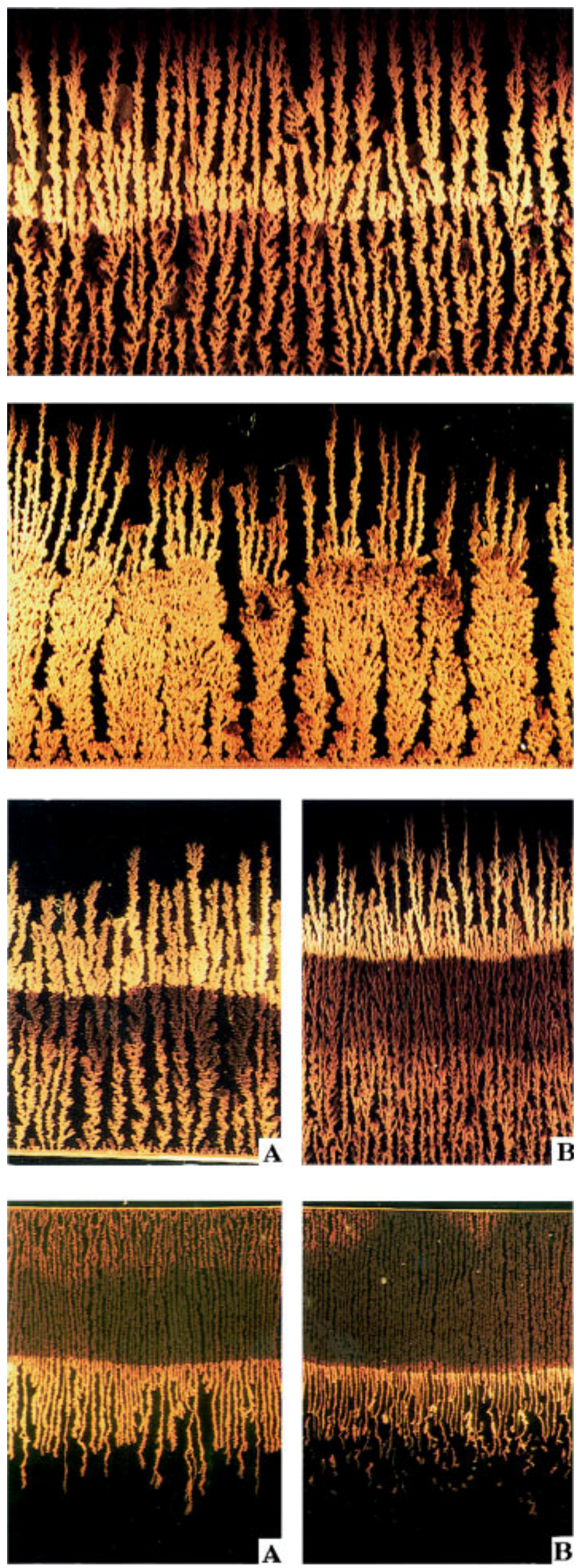

FIG. 1. (Color) Electrodeposit obtained from a $5 \cdot 10^{-2} M$ $\mathrm{CuSO}_{4}$ at $30 \mathrm{~V}$. Cell thickness, $300 \mu \mathrm{m}$; electrode separation, $4 \mathrm{~cm}$; deposit width, $35 \mathrm{~mm}$.
FIG. 2. (Color) Electrodeposit obtained from a $1 \cdot 10^{-1} M$ $\mathrm{CuSO}_{4}$ aqueous solution at $40 \mathrm{~V}$. Cell thickness, $300 \mu \mathrm{m}$; electrode separation, $4 \mathrm{~cm}$; deposit width, $35 \mathrm{~mm}$.
FIG. 3. (Color) Influence of the applied potential on the position of the transition. Electrodeposits obtained from a $5.10^{-2} \mathrm{M} \mathrm{CuSO}_{4}$ aqueous solution. Cell thickness, $300 \mu \mathrm{m}$; electrode separation, $4 \mathrm{~cm}$; deposit width, $35 \mathrm{~mm}$. (a) $30 \mathrm{~V}$ and (b) $60 \mathrm{~V}$.
FIG. 4. (Color) Electrodeposit obtained from a $1.10^{-1} M$ $\mathrm{CuSO}_{4}$ aqueous solution in a vertical cell with the cathode facing downward. Cell thickness, $300 \mu \mathrm{m}$; electrode separation, $4 \mathrm{~cm}$; deposit width, $35 \mathrm{~mm}$. (a) $60 \mathrm{~V}$ and (b) $105 \mathrm{~V}$. 
was mounted over a $2^{\circ}$ tilted $\mathrm{Si}(001)$ single crystal to improve the signal-to-noise ratio. If only a qualitative analysis was performed, a standard scan from $4^{\circ}$ to $70^{\circ} 2 \Theta$ with a step size of $0.05^{\circ} 2 \Theta$ and a measuring time of $3^{\prime \prime}$ /step was used. If, in addition, a quantitative analysis was required, a more detailed scan was measured from $25^{\circ}$ to $100^{\circ} 2 \Theta$ with a step size of $0.025^{\circ} 2 \Theta$ and a measuring time of $8^{\prime \prime} /$ step. The possibility of a partial oxidation of samples has been checked [8]. For this purpose, samples stored under argon atmosphere and under air have been analyzed and no significative differences have been found. Finally, the temporal evolution of the $\mathrm{x}$-ray-diffraction patterns also has been checked. In this case, although the intensity of all the peaks decreases slightly with time due to a loss of sample in successive manipulations, no significant changes in the composition of the deposit are obtained.

Electrolyte solutions were prepared with ultrapure water supplied by a Millipore-Milli $Q$ system. All chemicals were obtained from Merck quality p.a. and used as received.

\section{EXPERIMENTAL RESULTS AND DISCUSSION}

\section{A. General characteristics of transitions from binary copper sulphate solutions}

Dynamic morphological transitions in electrodeposits obtained from a nondeaerated copper sulphate solution are observed within the concentration range comprised between $0.01 M$ and $0.2 M$. The transition involves a change in the color and the ramification of the deposit. At low copper sulphate concentration $(0.05 M)$ (Fig. 1) the first deposit is reddish and dull and turns to a red and bright structure after the transition. At higher concentration values the difference in color diminishes and the transition is mostly evidenced as a change of the branching degree, which also depends on the experimental conditions (Fig. 2). These results are in broad agreement with previous reports from different research groups [1-3]. However, it was not previously reported that the position of the transition $\lambda$, defined as the distance from the transition to the cathode relative to the electrode separation, depends on the applied potential. Our experimental results show that, at low potential values $(\sim 20 \mathrm{~V})$, the values of $\lambda$ are close to 0.25 , but at higher potentials the transition is retarded and higher values of $\lambda$ are found (Fig. 3 and Table I). In a vertical cell with the cathode facing downward, the transition is also clearly defined and always appears at onefourth of the distance between the electrodes $(\lambda \simeq 0.25)$ (Fig. 4 ), independently of the applied potential.

This dynamic transition is also affected by the nature of the anion of the copper salt. The same behavior as described for copper sulfate solutions also has been observed using copper perchlorate solutions (Fig. 5), but the transition is far less well defined or no longer exists in copper acetate solutions. In the absence of oxygen in the electrolyte solution, the transition is also not clearly seen.

\section{B. Chemical composition of the electrodeposits}

The phase composition of the electrodeposits was determined by means of x-ray powder diffraction measurements. The samples that have been analyzed correspond to electrodeposits obtained in a 300- $\mu \mathrm{m}$-thick cell. Their qualitative

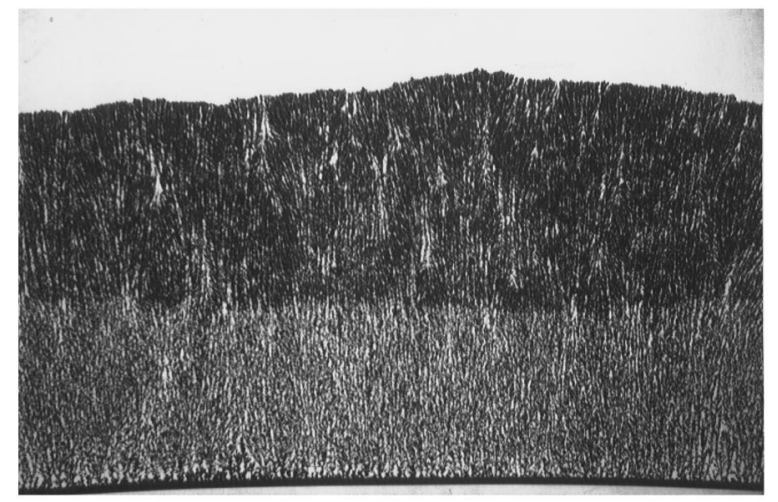

FIG. 5. Electrodeposit obtained from a $1.10^{-1} M \mathrm{Cu}\left(\mathrm{ClO}_{4}\right)_{2}$ aqueous solution at $40 \mathrm{~V}$. Cell thickness, $300 \mu \mathrm{m}$; electrode separation, $4 \mathrm{~cm}$; deposit width, $35 \mathrm{~mm}$.

analysis has been done by a comparison with the standard reference materials of the International Center for Diffraction Data data bank [9]. In all cases, a mixture of copper [powder diffraction file (PDF) 4-836] and cuprous oxide (PDF 5-667) has been identified. These results are shown in Fig. 6, which allows us to compare the plots of typical $\mathrm{x}$-ray data for samples collected before and after the morphological transition in the $2 \Theta$ range between $25^{\circ}$ and $100^{\circ}$. For both cases, peaks at the same $2 \Theta$ values $\left(43.30^{\circ}, 50.43^{\circ}, 74.13^{\circ}, 89.93^{\circ}\right.$, and $95.14^{\circ}$ for copper and $29.56^{\circ}, 36.42^{\circ}, 42.30^{\circ}, 52.46^{\circ}$, $61.35^{\circ}, 73.53^{\circ}, 77.33^{\circ}$, and $92.38^{\circ}$ for cuprous oxide) but with different intensities are observed. The quantitative analysis of these diffractograms has been made following two different procedures, which, however, lead to similar results. First, we employed the Rietveld method [10], namely, a profile fitting of the observed diffractogram; the phase composition of the mixture was determined from the scale factor ratio between the two components [11], copper and cuprous oxide, with fixed crystal structures. This method was applied by means of the FULLPROF $[12,13]$ program. Complementarily, we used the Chung method [14], which deals with the intensity ratios of a selected set of peaks and suitable calibration constants. Using both methods, we have obtained very similar compositions, close to within a $5 \%$ deviation. From this analysis we can conclude that the amount of cuprous oxide before the transition is higher $(\sim 42 \%)$ than after $(\sim 21 \%)$ the morphological change has occurred.

The presence of cuprous oxide in electrodeposits obtained from a copper sulphate solution can be justified assuming a competition between the two electrode reactions

$$
\begin{aligned}
2 \mathrm{Cu}^{2+}+2 \mathrm{OH}^{-}+2 e^{-} & \rightleftharpoons \mathrm{Cu}_{2} \mathrm{O}+\mathrm{H}_{2} \mathrm{O}, \\
\mathrm{Cu}^{2+}+2 e^{-} & \rightleftharpoons \mathrm{Cu} .
\end{aligned}
$$

At the $p \mathrm{H}$ values corrresponding to $0.05 \mathrm{M}$ copper sulphate solutions $(\sim 4.5)$, the first reaction can take place with a significant extent to give rise to a deposit with a substantial content of cuprous oxide. When the acid front meets the growing deposit, this reaction is hindered and the direct reduction of copper ions to metallic copper is enhanced. Thus the color change from a red but dull deposit to a bright and 

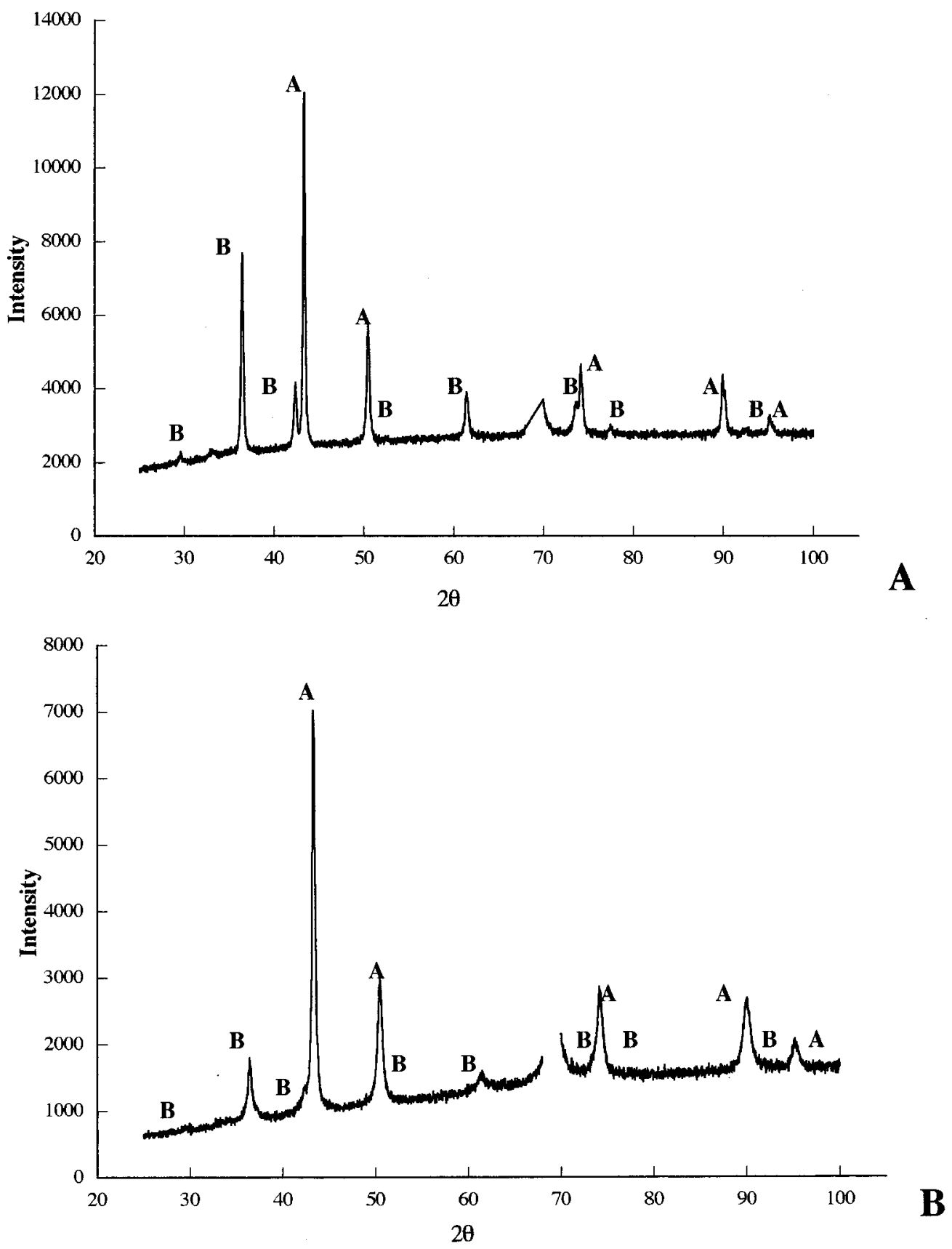

FIG. 6. Powder x-ray-diffraction patterns of electrodeposits obtained from a $5.10^{-2} M \mathrm{CuSO}_{4}$ aqueous solution at $30 \mathrm{~V}$ (a) before the transition $\left(42 \% \mathrm{Cu}_{2} \mathrm{O}\right)$ and (b) after the transition $\left(21 \% \mathrm{Cu}_{2} \mathrm{O}\right)$. (a) $\mathrm{Cu}$ and $\left(\right.$ b) $\mathrm{Cu}_{2} \mathrm{O}$.

more metallic structure can be interpreted according to this different chemical composition. Moreover, the different branching extent can be also understood in terms of a change in the resistivity of the deposit following the transition. Actually, it has been reported that an increase in the deposit resistivity favors the appearance of denser structures as a consequence of the diminution of screening effects inside the aggregate [15].

At concentrations of copper sulphate higher than $0.1 \mathrm{M}$, a red and bright deposit is always obtained and the transition is never observed. This admits a simple interpretation since the cuprous oxide content of the deposit, in this case, is too low (lower than $11 \%$ ) to evidence any change after meeting the anodic acid front. Conversely, at low copper sulphate con- centrations $(c \leqslant 0.01 M)$, where a dull reddish deposit is first obtained, the transition is also not observed. In this case, the low current values attained under such conditions trigger a small flux of protons associated with the anodic acid front that cannot decrease to a sufficient degree the cuprous oxide contents of the deposit (higher than 44\%).

The absence of the transition in experiments from an oxygen free copper sulphate solution and its poorly defined appearance from a copper acetate solution can be explained in terms of the $p \mathrm{H}$ values in the region close to the cathode surface and in the bulk solution, respectively. The initial $p \mathrm{H}$ values of copper acetate solutions are higher than for copper sulphate solutions $(p \mathrm{H} \sim 5.6$ at $0.05 M)$ and then, when the front meets the growing deposit, the $p \mathrm{H}$ is not reduced 
enough to cause the transition. On the other hand, the absence of oxygen reduction at the cathode diminishes the local $p \mathrm{H}$ at the cathode surface and a poorer cuprous oxide deposit $(\sim 23 \%)$ is obtained in a such a way that the acid front does not substantially affect its properties.

An extreme situation concerning this discussion on the chemical composition of such electrodeposits is worth mentioning at this point in relation to the finger instability observed in electrodeposition experiments from a copper sulphate solution in the presence of an inert electrolyte, typically any alcaline sulphate $[6,16,17]$. In this case, the inert electrolyte increases the solution conductivity and decreases the transference number of $\mathrm{Cu}^{2+}$, enhancing hydrogen evolution and therefore causing a local $p \mathrm{H}$ increase at the cathode surface. Then, the extent of the first reaction in (1) is largely favored and a much higher content of cuprous oxide is found in the deposit (up to 90\% [6]). This prompts the development of a completely different and unexpected morphology in the context of thin-layer electrodeposition, characterized by fingerlike modulated deposits with a very dense texture. Actually, such a cellular structure reflects the hydrodynamic instability undergone by the concentration layer encompassing the deposit and separating the bulk solution and the copper ion depleted solution embedding the electrodeposit. This instability is very similar to that responsible for the formation of viscous fingering in hydrodynamic systems. A detailed discussion can be found in Ref. [17].

\section{Experimental conditions for the Ohmic regime}

The electrodeposits obtained from a copper sulphate solution within the concentration and potential ranges here examined show the general features of the homogeneous morphology, i.e., a regular array of variably packed ramified filaments terminated at a growing front that reproduces the geometry of the cell. In these cases, it has been accepted that the growth velocity is controlled by ionic migration $[3,18]$ according to the small extent of the cathodic concentration layer [19]. In spite of this more or less broad agreement, we would like to accurately verify the validity of such an Ohmic regime at different growth stages. In a first step, this study is performed at initial stages of the experiment to minimize the contributions from the development of gravity-induced convection rolls [20-23] and temperature changes during growth due to heat dissipation. Afterward, this analysis is extended to comprise larger deposit developments.

For binary electrolytes, the assumption of a migration controlled growth leads to a close relation between the anion mobility $u_{-}$and the growth velocity $v[3,16,18]$ expressed as

$$
v=u_{-} E,
$$

where $E$ is the applied electric field. Then we can compare sulphate mobility values obtained in electrodeposition experiments at initial stages (Table II) with those derived from conductivity measurements and transference numbers [24] equal to $2.75 \times 10^{-4}$ and $2.55 \times 10^{-4} \mathrm{~cm}^{2} \mathrm{~s}^{-1} \mathrm{~V}^{-1}$ for $0.05 \mathrm{M}$ and $0.1 M$ copper sulphate solutions, respectively. It is worth emphasizing that very good agreement is achieved especially at high concentrations and applied potentials. This can be interpreted since under such conditions the deposits are
TABLE I. Position of the transition ( $\lambda$ values) vs applied potential. The electrode separation is $4 \mathrm{~cm}$ and the cell thickness is 300 $\mu \mathrm{m}$.

\begin{tabular}{rcc}
\hline \hline$\Delta V / V$ & $5 \times 10^{-2} M \mathrm{CuSO}_{4}$ & $1 \times 10^{-1} M \mathrm{CuSO}_{4}$ \\
\hline 20 & 0.25 & 0.25 \\
30 & 0.25 & 0.25 \\
40 & 0.25 & 0.30 \\
50 & 0.25 & 0.30 \\
60 & 0.28 & 0.33 \\
70 & 0.30 & 0.35 \\
80 & 0.33 & 0.35 \\
90 & 0.33 & 0.38 \\
100 & 0.38 & 0.43 \\
110 & 0.38 & \\
120 & 0.43 & \\
\hline \hline
\end{tabular}

denser and more space filling, thus making the hypothesis behind an Ohmic model mostly fulfilled $[16,18,24]$. In passing, we remark that the agreement evidenced here indicates that quasi-two-dimensional electrodeposition experiments may be considered a useful approach to estimate mobility values of ions as also proposed previously by Kuhn and Argoul [25].

The validity of the Ohmic control during later stages of the deposit development has been checked also studying the temporal evolution of the growth velocity (Fig. 7). Their experimental values are compared with those calculated from

$$
v=u_{-} \frac{\Delta V}{d-l(t)}
$$

where $d$ is the electrode separation, $l(t)$ stands for the front position, and $u_{-}$is the anion mobility obtained from measurements at initial conditions. Significant discrepancies between both sets of values are detected at long times. We can observe that experimental values are larger than theoretical ones, mainly at high potentials. This can be interpreted assuming that other transport regimes, different from migration, would play an important role in the whole electrodeposition process. In particular, the existence of convection currents has been reported previously in thin-layer cells [2023]. Such a convective pattern, organized in convection rolls, is due to the existing density gradients in the anodic and cathodic concentration layers. Such rolls first propagate from

TABLE II. Sulphate mobilities measured from electrodeposition experiments. The electrode separation is $4 \mathrm{~cm}$ and the cell thickness is $300 \mu \mathrm{m}$.

\begin{tabular}{lccc}
\hline \hline$\Delta V / V$ & $\begin{array}{c}u_{-} \times 10^{-4} \mathrm{~cm}^{2} \mathrm{~s}^{-1} \mathrm{~V}^{-1} \\
5.10^{-2} \mathrm{M} \mathrm{CuSO}_{4}\end{array}$ & $\Delta V / V$ & $\begin{array}{c}u_{-} \times 10^{-4} \mathrm{~cm}^{2} \mathrm{~s}^{-1} V^{-1} \\
1.10^{-1} \mathrm{M} \mathrm{CuSO}_{4}\end{array}$ \\
\hline 60 & 2.66 & 60 & 2.51 \\
50 & 3.17 & 50 & 2.37 \\
40 & 3.33 & 40 & 2.42 \\
30 & 3.70 & 30 & 2.63 \\
& & 20 & 3.06 \\
& & 10 & 4.07 \\
\hline \hline
\end{tabular}




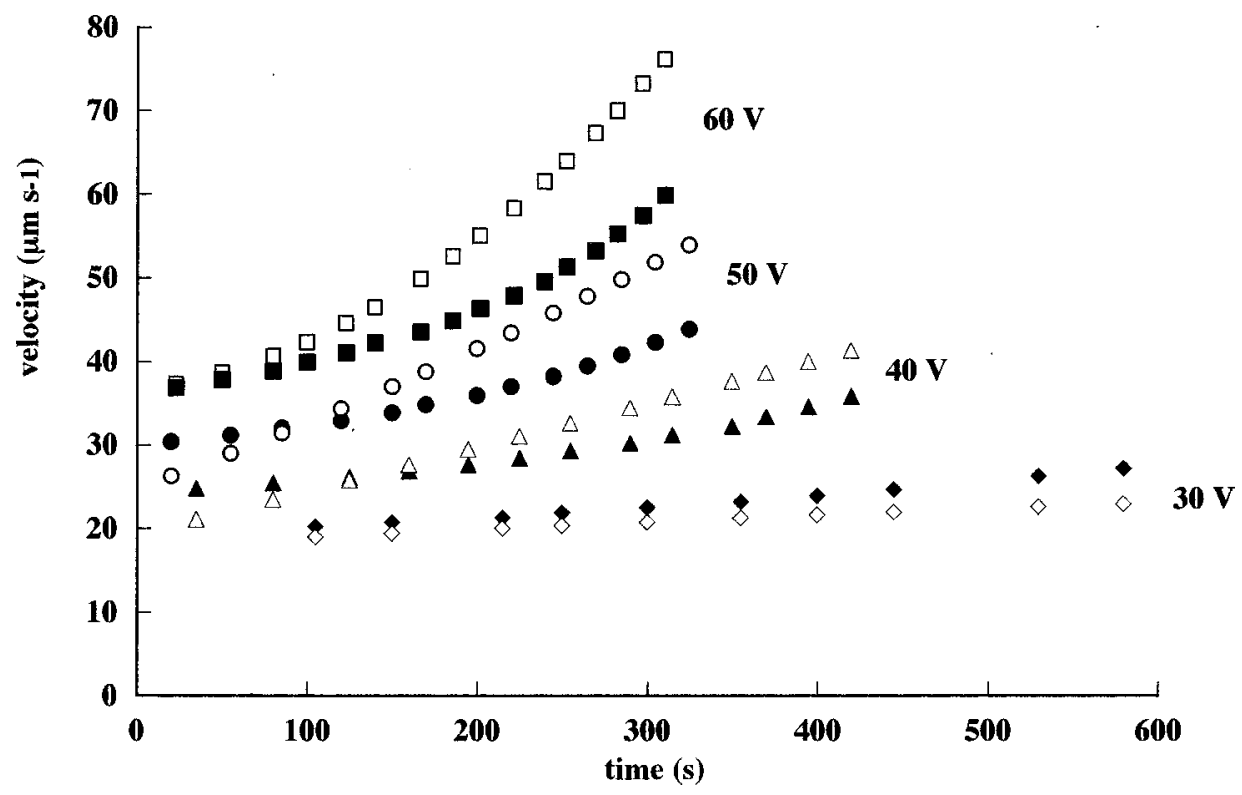

FIG. 7. Temporal dependence of growth velocity of electrodeposits obtained from a $1.10^{-1} M \mathrm{CuSO}_{4}$ aqueous solution at different potentials. Open symbols, experimental values; solid symbols, values obtained by Eq. (3).

both electrodes to the middle of the cell, where they meet and collapse into a large-scale flow encompassing the whole solution. Different dependences of the roll size on experimental parameters have been proposed. Chazalviel et al. [23] recognize two different regimes: At short times, the width of the roll $L$ scales with curent density $J$ and time as

$$
L \propto J^{3 / 10} t^{4 / 5}
$$

whereas the long-time behavior is more accurately described by

$$
L \propto J^{1 / 3} t^{1 / 2}
$$

Similar temporal dependences have been also proposed by Huth et al. [22]. They also suggest that the long-time behavior is the most relevant regime in typical electrodeposition experiments.

In particular, two effects can be attributed to the cathodic roll: (i) an increase of the amount of copper ions reaching the cathode surface from the bottom of the cell and (ii) an increase of the velocity of the anions leaving the cathodic region. These two contributions can explain the higher experimental values of $v$ as compared to the theoretically predicted ones (Fig. 7). In view of the dependence of $L$ on the current density (5), this effect would be singularly relevant at high potentials, as we find in our experiments. Another consequence of the existence of such convection currents will be discussed in the following subsection.

\section{Position of the dynamical transition and proton mobility}

As mentioned above, a morphological transition takes place when the growing deposit first meets a proton front originated in the hydrolysis of the dissolved copper ions at the anode [1-3]. Then, assuming an Ohmic control for the whole electrochemical process, the position of the transition $\lambda$ will depend on the relative values of the growth velocity of the deposit and the velocity of the anodic front. This in turn could be expressed in terms of the relative mobilities of the hydrogen ion $u_{\mathrm{H}}$ and the anion of the copper salt $u_{-}$that limits the velocity of the growing deposit. A straightforward calculation leads to the following value for $\lambda$ :

$$
\lambda=\frac{u_{-}}{u_{-}+u_{\mathrm{H}}} .
$$

As mentioned in Sec. III A, the values of $\lambda$ obtained in a vertical cell with the cathode facing downward are around 0.25 . In these conditions, the absence of significant contributions of gravity-induced convection effects during the deposit growth allows us to assume that Ohmic conditions are mostly reproduced. Then Eq. (6) leads to a value of proton mobility around $7.5 \times 10^{-4} \mathrm{~cm}^{2} \mathrm{~s}^{-1} \mathrm{~V}^{-1}$ in $0.05 M$ copper sulphate solutions. This value is much lower than proton mobility evaluated in an acid solution at similar values of ionic strength $\left(\sim 2.5 \times 10^{-3} \mathrm{~cm}^{2} \mathrm{~s}^{-1} \mathrm{~V}^{-1}\right.$ in sulphuric acid and $\sim 3.3 \times 10^{-3} \mathrm{~cm}^{2} \mathrm{~s}^{-1} \mathrm{~V}^{-1}$ in hydrochloric acid solutions [26]) and the values calculated by means of the DebyeHückel-Onsager theory for an ionic strength equal to $0.2 \mathrm{M}$ $\left(3.1 \times 10^{-3} \mathrm{~cm}^{2} \mathrm{~s}^{-1} \mathrm{~V}^{-1}\right)$ [27].

These low values of the proton mobility in the presence of copper ions might be interpreted considering the two contributions to the transport mechanism of hydrogen ion in aqueous solutions [28]: the hydrodynamic transfer, common to all ions, and the prototropic effect, as a consequence of successive proton transfers between $\mathrm{H}_{3} \mathrm{O}^{+}$and $\mathrm{H}_{2} \mathrm{O}$. Actually, this last mechanism is mainly responsible for the anomalously high conductivity values of hydrogen ions. It is then manifestly clear that the proton mobility will depend on the structure of water and that any factor affecting this structure will also modify the proton mobility. In this sense, ionic effects on water structure and subsequently on proton mobility in aqueous solutions have been reported in the literature long ago [28-30]. These studies are basically concerned with the influence of monovalent cations such as lithium salts. In particular, it is well known that this ion markedly decreases the 
proton mobility in aqueous media as a consequence of changes in the water structure due to electrostatic interactions. More recently, ion dynamics in aqueous electrolytes has been investigated by means of high-resolution quasielastic neutron scattering [31]. Using this experimental approach, proton diffusion in aqueous solutions in the presence of divalent cations such as copper and nickel has been studied [32]. The experimental results show that the value of the average diffusion coefficient of all water protons in concentrated $\mathrm{Cu}^{2+}$ solutions $(3 \mathrm{~m})$ at room temperature is around $10^{-5} \mathrm{~cm}^{2} \mathrm{~s}^{-1}$. Using this estimate, the Einstein equation leads to a rough value of proton mobility around $8 \times 10^{-4} \mathrm{~cm}^{2} \mathrm{~s}^{-1} \mathrm{~V}^{-1}$. In spite of the different concentration range of our experiments and those just reported, the close agreement existing between this value of proton mobility and that obtained by measuring the position of the acid morphological transition occurring in our electrochemical cell $\left(7.5 \times 10^{-4} \mathrm{~cm}^{2} \mathrm{~s}^{-1} \mathrm{~V}^{-1}\right)$ is remarkable. Once more this indicates the sensitivity of the growth parameters in thin-layer electrodeposition experiments with respect to ionic mobilities.

In contrast, we recall at this point that in a horizontal cell the values of $\lambda$ increase with the applied potential at high potential values. Since this behavior has not been observed in a vertical cell with the cathode facing downward where a constant value of $\lambda$ is obtained, we can conclude that the gravity-induced convection rolls [20-23], discussed the Sec. III C, may also play a role in the onset of such morphological transitions. Actually, a dynamical transition different from the proton originated one discussed so far has been identified to take place when the anodic convection roll meets the growing deposit during zinc electrodeposition [22].

In our horizontal cell, the position of the transition is retarded, namely, the velocity of the acid front is decreased compared to that of the growing deposit, when the potential increases at high potential values. Under these experimental conditions and taking into account the convection roll dynamics [see Eqs. (4) and (5)], the acid front is expected to reach the growing deposit before the anodic roll [23]. This rules out that the transition might really be associated with the meeting of the growing deposit and the anodic roll.

However, the nature itself of such convection currents and our own experimental observations of the way they modify a pure Ohmic control provide us with some clues to interpret the observed delay in the proton-originated morphological transition. First of all, we have already verified that the deposit velocity is larger in the presence of such convection currents. In addition, the protons originated near the anode can be at least initially somehow trapped and, in turn, their motion hindered by the anodic roll, so that a transient decrease of the proton transport rate induced by such convection currents cannot be completely ruled out.

\section{CONCLUDING REMARKS}

The morphological dynamic transitions observed in thinlayer electrodeposition experiments from a binary copper sulphate solution arise as a consequence of the competition between the formation of metallic copper and cuprous oxide during the whole reduction process. The presence of cuprous oxide leads to a dull and reddish deposit that can turn red and bright after meeting the acid front originated in the anodic reactions. This change mainly occurs when the amount of cuprous oxide in the deposit is neither too high nor too small to be insensitive to the change in the chemical composition of the solution near the growing front.

The position of the transition is mainly due to the migration rates of the sulphate anion, which determines the growth velocity, and the hydrogen ion, responsible for the chemical front coming from the anodic region. However, nonnegligible contributions of the gravity-induced convection rolls also have to be taken into account. When convection effects are present, sensible deviations from a purely ionic migration controlled process are clearly evidenced mainly for long times and high applied potentials. However, at initial stages, when the convection effect should not be relevant, the agreement between the sulphate mobility values obtained in our experiments and those calculated from conductivity measurements is remarkable. In contrast, the values of the proton mobility derived from the position of the transition in a vertical cell, i.e., in the absence of convection effects, are very low, but qualitatively agree with values obtained using different experimental techniques in similar conditions.

\section{ACKNOWLEDGMENTS}

M.Q.L.S. benefited from a grant from Ministerio de Educación y Ciencia (Spain). Financial support from Dirección General de Investigación Científica y Tecnológica (Spain) under Project No. PB93-0759 is acknowledged.
[1] P. Garik, D. Barkey, E. Ben-Jacob, E. Bochner, N. Broxholm, B. Miller, B. Orr, and R. Zamir, Phys. Rev. Lett. 62, 2703 (1989).

[2] J. R. Melrose, D. B. Hibbert, and R. C. Ball, Phys. Rev. Lett. 65, 3009 (1990).

[3] V. Fleury, M. Rosso, and J. N. Chazalviel, Phys. Rev. A 43, 6908 (1991).

[4] P. P. Trigueros, J. Claret, F. Mas, and F. Sagués, J. Electroanal. Chem. 312, 219 (1991).

[5] M. A. Guzman, R. D. Freimuth, P. U. Pendse, M. C. Veinott, and L. Lam, in Nonlinear Structures in Physical Chemistry, edited by L. Lam and H. C. Morris (Springer, New York, 1990), p. 32.

[6] M.-Q. Lòpez-Salvans, F. Sagués, J. Claret, and J. Bassas, J. Electroanal. Chem. 421, 205 (1997).

[7] A. Kuhn and F. Argoul, Phys. Rev. E 49, 4298 (1994).

[8] K. Mori, Y. Okai, H. Horie, and H. Yamada, Corros. Sci. 32, 1237 (1991).

[9] Powder diffraction file, PDF-2, 1995, International Center for Diffraction Data, 12 Campus Boulevard, Newton Square, PA 19073-3273.

[10] H. M. Rietveld, J. Appl. Crystallogr. 2, 65 (1969). 
[11] D. L. Bisch and S. A. Howard, J. Appl. Crystallogr. 21, 86 (1988).

[12] J. Rodriguez-Carvajal, FULlPRoF: A Program for Rietveld Refinement and Pattern Matching Analysis, Abstracts of the Satellite Meeting on Powder Diffraction of the XV Congress of the IUCr., Toulouse, 1990 (International Union of Crystallography, Toulouse, 1990), p. 127.

[13] J. Rodriguez-Carvajal, FULLPROF version 3.0.0, unpublished software, Laboratoire Leon Brillouin, CEA-CNRS, 1990.

[14] F. H. Chung J. Appl. Crystallogr. 7, 526 (1974).

[15] C. P. Chen and J. Jorné, J. Electrochem. Soc. 137, 2047 (1990).

[16] P. P. Trigueros, F. Sagués, and J. Claret, Phys. Rev. E 49, 4328 (1994).

[17] M.-Q. Lòpez-Salvans, P. P. Trigueros, S. Vallmitjana, J. Claret, and F. Sagués, Phys. Rev. Lett. 76, 4062 (1996).

[18] D. Barkey, P. Garik, E. Ben-Jacob, B. Miller, and B. Orr, J. Electrochem. Soc. 139, 1044 (1992).

[19] J. Mach, F. Mas, and F. Sagués, Europhys. Lett. 25, 271 (1994).

[20] D. P. Barkey, D. Watt, Z. Liu, and S. Raber, J. Electrochem. Soc. 141, 1206 (1994).
[21] M. Rosso, J. N. Chazalviel, V. Fleury, and E. Chassaing, Electrochim. Acta 39, 507 (1994).

[22] J. M. Huth, H. L. Swinney, W. D. McCormick, A. Kuhn, and F. Argoul, Phys. Rev. E 51, 3444 (1995).

[23] J. N. Chazalviel, M. Rosso, E. Chassaing, and V. Fleury, J. Electroanal. Chem. 407, 61 (1996).

[24] D. G. Miller, J. A. Rard, L. B. Epstein, and R. A. Robinson, J. Solution Chem. 9, 467 (1980).

[25] A. Kuhn and F. Argoul, J. Chem. Educ. 71, A273 (1994).

[26] V. M. M. Lobo, Handbook of Electrolyte Solutions (Elsevier, Amsterdam, 1989), Pt. A, p. 664.

[27] J. O'M. Bockris and A. K. N. Reddy, Modern Electrochemistry (Plenum, New York, 1977), Vol. 1.

[28] S. Lengyel and B. E. Conway, Comprehensive Treatise of Electrochemistry (Plenum, New York, 1983), Vol. 5, Chap. 4, p. 339.

[29] L. A. Woolf, J. Phys. Chem. 60, 481 (1960).

[30] D. Glietenberg, A. Kutschker, and M. V. Stackelber, Ber. Bunsenges. Phys. Chem. 72, 562 (1968).

[31] P. S. Salmon, J. Phys. C 20, 1573 (1987).

[32] P. S. Salmon, W. S. Howells, and R. Mills, J. Phys. C 20, 5727 (1987). 